\title{
Unicity in the uniform approximation of vector-valued functions
}

\author{
Lee W. Johnson
}

\begin{abstract}
The problem of unicity in the uniform approximation of vector-valued functions is considered. A recent result of Cheney and Wulbert concerning unicity in the uniform approximation of real-valued functions is extended and some "point-wise" criteria for unicity are given.
\end{abstract}

\section{Introduction}

Using gauge functions, the classical theory of uniform approximation of real functions of a real variable can be generalized in a natural fashion to cover functions which assume values in $n$-dimensional Euclidean space. In particular, let $X$ be a compact Hausdorff space and let $C(X)$ be the set of continuous functions from $X$ to $E^{n}$. Suppose $U$ is a closed, bounded and convex subset of $E^{n}$ with non-empty interior. Let the origin $\theta$ be in $U$ (although not necessarily in the interior of $U$ ) and let $G$ be the gauge function for $U$

$$
G(y)=\inf \{a \mid y \in a U, a \geq 0\}
$$

where we set $G(y)=\infty$ if $y$ is in no multiple of $U$.

A functional $M$ can be defined on $C(X)$ by

$$
M(f)=\sup \{G(f(x)) \mid x \in X\}
$$

where $M(f)=\infty$ if no multiple of $U$ absorbs the range of $f$. If $P$ is a linear subspace of $C(X)$, we call $p \in P$ an approximation to $f$ if 
$M(p-f)<\infty$ and a best uniform approximation if $M(p-f) \leq M(q-f)$ for all $q$ in $P$. If $U$ is symetric, then $G$ defines a norm on $E^{n}$. This makes $C(X)$ a normed linear space where $M(f)$ is the usual sup norm on $C(X)$. However, generalizations of many of the classical theorems can be established from the convexity of $U$, so assuming $C(X)$ is normed is unduly restrictive.

In [4], the author gave several characterizations of best approximations to vector-valued functions together with some applications. These were obtained by using extreme points and by extending the concept of $H$-sets which was introduced by Collatz in [3]. In this paper we consider problems of unicity in approximating vector-valued functions. Theorem 3, for example, gives both a simple proof and an extension of a recent result of Cheney and Wulbert [1] characterizing those subspaces of $C(X)$ in which best approximations are unique when the elements of $C(X)$ are real functions.

It should be noted that some of the results of [4] can be extended to functions with values in a topological vector space should such an extension be useful.

\section{The extreme points}

Let $p$ be an approximation to $f$, with $M(p-f)=a$. Let

$$
E(p, f)=\{x \mid[p(x)-f(x)] \in \partial(a U)\}
$$

where $\partial(U)$ is the topological boundary of $U$. We call $E(p, f)$ the set of extreme points of $p-f$.

We need the following results, which may be found in [4]. The functional $G$ satisfies

$$
\begin{aligned}
& G(y) \geq 0, G(\theta)=0, \\
G(t y)= & t G(y) \text { for all real } t, t \geq 0, \\
& G(y+z) \leq G(y)+G(z) .
\end{aligned}
$$

The set of best approximations to $f$ by functions in $P$ is a convex set. The functional $G$ is continuous on $\{y \mid G(y)<\infty\}$ if and only if the set $U_{1}=\{y \mid G(y)=1\}$ is closed in $E^{n}$. We assume hereafter that $U_{1}$ is 
closed. Finally, we have the following characterization theorem.

THEOREM 1. $p$ is a best approximation to $f$ if and only if $p$ is best on $E(p, f)$.

By $p$ best on $E(p, f)$ we mean: if $q$ is any approximation to $f$ then

$$
\sup \{G(p(x)-f(x)) \mid x \in E(p, f)\} \leq \sup \{G(q(x)-f(x)) \mid x \in E(p, f)\} .
$$

Theorem 1, together with Theorem 2 below, will be used to derive the results of the next section.

We call $U$ strictly convex if when $y$ and $z$ are distinct points in $\partial(U)$ then $\lambda y+(1-\lambda) z$ is in the interior of $U$ for $0<\lambda<1$.

THEOREM 2. Let $U$ be strictly convex and let $p$ and $q$ be best approximations to $f$. Let $h=\lambda p+(1-\lambda) q, 0<\lambda<1$. Then, $x \in E(h, f)$ if and only if $x \in E(p, f) \cap E(q, f)$ and $p(x)=q(x)$.

Proof. If $M(p-f)=M(q-f)=a$ then $M(h-f)=a$ since the set of best approximations is convex. If $x$ is in $E(h, f)$ then $\lambda p(x)+(1-\lambda) q(x)-f(x)=\lambda[p(x)-f(x)]+(1-\lambda)[q(x)-f(x)]$ is in $\partial(a U)$. By assumption, $[p(x)-f(x)] \in a U$ and $[q(x)-f(x)] \in$ all. If $p(x)-f(x)$ were in the interior of $a U$ then so would be the entire line segment joining $p(x)-f(x)$ and $q(x)-f(x)$ with the possible exception of $q(x)-f(x)$ (cf. [2]). Hence, $x \in E(p, f) \cap E(q, f)$. Since $\lambda[p(x)-f(x)]+(1-\lambda)[q(x)-f(x)]$ is in $\partial(a U)$ it follows by strict convexity that $p(x)=q(x)$. The converse is immediate.

UNICITY. Let 0 denote the function defined by $0: x \rightarrow \theta$ for all $x$ in $X$. Following Cheney and Wulbert, we say a subset $A$ of $X$ is an a-set if there is a function $f$ in $C(X)$ such that $A=E(0, f)$ where 0 is a best approximation to $f$. We say $p$ vanishes on $A$ if $p(x)=\theta$ for each $x$ in $A$. The following is an extension of Theorem 10 in [1].

THEOREM 3. Let $U$ be strictly convex. Each function in $C(X)$ has at most one best approximation if and only if 0 is the only function in $P$ vanishing on an $\alpha-s e t$.

Proof. Suppose $f \in C(X)$ and suppose $p$ and $q$ are best approximations to $f$. Form $h=\frac{1}{2} p+\frac{1}{2} q$ and note that $p-q$ vanishes 
on $E(h, f)$ by Theorem 2. But $E(h, f)=E(0, h-f)$ is an $\alpha$-set.

Conversely, suppose all best approximations are unique. Suppose $q \in P$ with $q(x)=\theta$ for all $x$ in $E(0, f)$, where $E(0, f)$ is an $\alpha$-set. Without loss of generality we may assume $M(f)=1$ and $M(q)<1$ for $M(\lambda f)=\lambda M(f)$ for all real $\lambda, \lambda \geq 0$. Form $\varphi(x)=[1-G(q(x))] f(x)$. We will show that $\lambda q$ is a best approximation to $\varphi$ for $0 \leq \lambda \leq 1$. Using (4),

$$
G(\varphi(x)-\lambda q(x)) \leq[1-G(q(x))] G(f(x))+\lambda G(q(x)) \leq 1-(1-\lambda) G(q(x)) \leq 1,
$$

for all $x$ in $X$. If there is $r \in P$ such that $M(\varphi-r) \leq 1-t$, $t>0$, then for all $x$ in $E(0, f)$ we have $G(\varphi(x)-r(x)) \leq 1-t$. Hence, $G(f(x)-r(x)) \leq 1-t$ for $x \in E(0, f)$ which is a contradiction, for 0 is the best approximation to $f$ and is hence best on $E(0, f)$. Thus, $M(\varphi-r) \geq 1$ for all $r \in P$ which means $\lambda q$ is a best approximation to $\varphi$ for $0 \leq \lambda \leq 1$, contradicting the supposed unicity of all best approximations.

Since it would appear to be a relatively rare happening that best approximations to all functions in $C(X)$ are unique, it is of some interest to have "point-wise" criteria for unicity. The following is a sufficient condition that a best approximation be unique.

THEOREM 4. Let $U$ be strictly convex and let $p$ be a best approximation to $f \cdot p$ is unique if 0 is the only function in $P$ vanishing on any a-set in $E(p, f)$.

We omit the proof of this theorem as it is exactly the same as the proof of the first part of Theorem 3. The hypothesis of strict convexity may be dropped from the next theorem.

THEOREM 5. Let $p$ be a best approximation to $f$; then $p$ is unique if and only if $p$ is unique on some open set containing $E(p, f)$.

Proof. Let $q$ be any approximation to $f$ and suppose $p$ is unique on $Q$, where $Q$ is open and $E(p, f) \subset Q$. Then

$$
M(p-f)=\sup _{x \in Q}\{G(p(x)-f(x))\}<\sup _{x \in Q}\{G(q(x)-f(x))\} \leq M(q-f) .
$$

Thus, $p$ is unique. 
Conversely, let $M(p-f)=a$ and suppose there is an approximation $q \in P$ such that $\sup _{x \in Q}\{G(q(x)-f(x))\} \leq M(p-f)$, where $Q$ is any open set containing $E(p, f)$. If $x \notin E(p, f)$ then $p(x)-f(x)$ is in the interior of $a U$. Therefore, for each $x$ in $X-Q$ there is $\lambda$, $0<\lambda<1$, such that $y=(1-\lambda)[p(x)-f(x)]+\lambda[q(x)-f(x)]$ is in the interior of $a U$ and thus $G(y)<a$.

Suppose there is no fixed $\lambda>0$ such that

$$
G((1-\lambda)[p(x)-f(x)]+\lambda[q(x)-f(x)]) \leq M(p-f)
$$

for all $x$ in the compact set $X-Q$. Then for every $n$ there is $x_{n} \in X-Q$ such that $G\left((1-1 / n)\left[p\left(x_{n}\right)-f\left(x_{n}\right)\right]+1 / n\left[q\left(x_{n}\right)-f\left(x_{n}\right)\right]\right)>M(p-f)$. We may as well suppose $\left\{x_{n}\right\} \rightarrow x_{0}, x_{0} \in X-Q$. The continuity of $G$ will imply $G\left(p\left(x_{0}\right)-f\left(x_{0}\right)\right) \geq M(p-f)$ which cannot be as $p\left(x_{0}\right)-f\left(x_{0}\right)$ is in the interior of all. Thus, there is some $\lambda^{\prime}, 0<\lambda^{\prime}<1$, for which (5) holds for all $x$ in $X-Q$.

$$
G(q(x)-f(x)) \leq M(p-f) \text { and } G(p(x)-f(x)) \leq M(p-f) \text { for all } x \text { in }
$$
$Q$, so by (4) we have (5) holding for all $\lambda, 0<\lambda<1$ when $x \in Q$. Thus, $\left(1-\lambda^{\prime}\right) p+\lambda^{\prime} q$ is a best approximation to $f$, contradicting the supposed unicity of $p$.

\section{References}

[1] E:W. Cheney and D.E. Wulbert, "The existence and unicity of best approximations", Math. Scand. 24 (1969), 113-140.

[2] H.G. Eggleston, Convexity (Cambridge Tracts in Mathematics and Mathematical Physics, no. 47. Cambridge University Press, Cambridge, 1958).

[3] L. Collatz, "Inclusion theorems for the minimal distance in rational Tschebyscheff approximation with several variables", Proc. Sympos. Genexal Motors Res. Lab. (1964), 43-56. Approximation of functions, edited by Henry L. Garabedian (Elsevier Publishing Co., Amsterdam, London, New York, 1965). 
[4] Lee W. Johnson, "Uniform approximation of vector-valued functions", Numer. Math. 13 (1969), 238-244.

Virginia Polytechnic Institute and State University, Blacksburg, Virginia, USA. 